\title{
The Resilience of Massive MIMO PNC to Jamming Attacks in Vehicular Networks
}

\author{
Bismark Okyere $^{\circledR}$, Graduate Student Member, IEEE, Leila Musavian ${ }^{\circledR}$, Member, IEEE, \\ Berna Özbek ${ }^{\circledR}$, Senior Member, IEEE, Sherif A. Busari ${ }^{\circledR}$, and Jonathan Gonzalez
}

\begin{abstract}
In this article, we investigate the resilience of Massive MIMO Physical Layer Network Coding (PNC) to jamming attack in both sub-6 $\mathrm{GHz}$ and millimeter-Wave (mmWave) systems in vehicular networks. Massive MIMO generally is resilient to jamming attacks, and we investigate the impact that PNC has on this resilience, if combined with Massive MIMO. The combination of Massive MIMO and PNC has shown a significant improvement in the bit error rate (BER) in our previous investigation. The corresponding framework is analysed against a barraging attack from a jammer, where the jamming channel is not known to the base station (BS), and the jammer can use any number of transmit antennas. Over Rayleigh channel, our simulation results reveal that Massive MIMO PNC performs better in the lower signal-to-noise ratio (SNR) regions to jamming attacks and this is achieved at twice the spectral efficiency. A similar performance is observed over mmWave channel.
\end{abstract}

Index Terms-Massive MIMO, physical layer network coding, vehicular networks, jamming.

\section{INTRODUCTION}

$\mathbf{T}$ HE broadcast nature of the wireless medium has undoubtedly propelled some significant innovations that allow users to access network resources from nearly any convenient location. These innovations enable mobile users to access real-time information whilst on the move, allowing increase in coverage and scalable deployment of network resources. Interference has been one of the main challenges to deal with in such an open air interface in wireless communication, where signals from multiple sources arrive simultaneously at a receiver. Although, there is no interference that is friendly, some forms of interference can be controlled by regulating the transmission of the interference sources. Unfortunately, some forms of interference are intentional and uncontrollable. They fall into the category of denial-of-service (DoS) attack and are often referred to as radio-frequency (RF) jamming.

Manuscript received March 3, 2020; revised June 8, 2020; accepted July 15, 2020. Date of publication September 14, 2020; date of current version July 12, 2021. This work was supported by the European Union Horizon 2020, RISE 2018 scheme (H2020-MSCA-RISE-2018) under the Marie SkłodowskaCurie under Grant 823903 (RECENT). The Associate Editor for this article was S. Mumtaz. (Corresponding author: Bismark Okyere.)

Bismark Okyere and Leila Musavian are with the School of Computer Science and Electronic Engineering, University of Essex, Colchester CO4 3SQ, U.K. (e-mail: bismark.okyere@essex.ac.uk; leila.musavian@essex.ac.uk).

Berna Özbek is with the Electrical-Electronics Engineering Department, İzmir Institute of Technology, 35430 İzmir, Turkey (e-mail: bernaozbek@ iyte.edu.tr).

Sherif A. Busari and Jonathan Gonzalez are with GS-Lda, 3750101 Àgueda, Portugal (e-mail: sbusari@gs-lda.com; jonathan@gs-lda.com).

Digital Object Identifier 10.1109/TITS.2020.3016907
RF jamming is a malicious attempt to overwhelm a wireless communication system with the objective of sabotaging the normal operation of the network [27].

Physical Layer Network Coding (PNC) is a key physical layer (PHY) technique that has the potential to overcome the adverse impacts of interference by applying Network Coding (NC) to received electromagnetic (EM) radio waves that constitute a superposition of a multitude of transmitted signals. The concept of PNC has been extensively studied in [1]-[3], among others. The original idea of applying NC at the PHY, leveraging on the additive nature of the wireless medium was detailed in [1], [2] where an information theoretic approach was employed, with emphasis on the rate region of the Gaussian two-way relay channel (TWRC). Joint channel coding and PNC was also investigated in [2] and the performance found to be better than just PNC.

MIMO is increasingly gaining attention in the PNC field, leveraging on its multiplexing gain. In [4], the authors proposed a linear detection based scheme using log-likelihood ratio (LLR) and selective combining. The relay utilizes the summation and difference of the two end packets, and then converts them to a NC symbol. The investigation in [4] employed a $2 \times 2$ MIMO with BPSK modulation. This work was then extended in [5] to a $4 \times 4$ MIMO relay system, where the relay is equipped with four antennas and two UEs, each equipped with two antennas. The authors observed a multiplexing gain when the number of antennas at both the relay node and the two UEs are increased. In [6] and [7], an eigen-direction alignment precoding scheme is proposed for MIMO TWRCs, where multiple independent PNC streams are created over aligned eigenmodes. In [8]-[10], analog network coding (ANC) based MIMO TWRC was investigated. However, ANC is known to propagate noise from one node to another, and therefore, the performance is not as good as the schemes in which each node tries to clean up the noise [11].

Later in [12]-[14], it was shown that in a MIMO PNC scheme, when a user selects the transmit antenna with the strongest channel condition, it significantly outperforms space-time block codes. A channel-quantized PNC (CQ-PNC) that converts $K$ received signals at the relay into two signals by an orthogonal matrix, Q, and upper triangular matrix, R, or QR matrices decomposition in a MIMO TWRC is proposed in [15] which showed that CQ-PNC can achieve full diversity gain of $K$. A full-duplex TWRC in Massive MIMO together with a lattice-based PNC was investigated in [16], which showed 
that their proposed scheme requires just a single time slot to exchange information across TWRC, whereas four time slots would be needed in a conventional TWRC. PNC has also been considered in 5G MIMO systems for backhauling, as opposed to cloud radio access network (Cloud-RAN), and also for coordinated multipoint (CoMP) [17]-[19].

Jamming attack in itself is considered under the umbrella of active attack in physical layer security (PLS) [20]. The broadcast nature of the wireless medium makes the physical layer the most vulnerable layer. PLS solutions approach security issues from information theoretic perspective, by leveraging on the randomness, interference and other characteristics as observed by the PHY. However, some attacks, particularly those categorised under active attacks, might require countermeasure approaches that include PHY algorithms and techniques that can withstand the attack with little or no degradation. The countermeasure approaches address the proactive types of jamming attacks [21], where the attacker's main objective is to thwart the normal operation of a wireless system by persistently sending jamming signals. One of the most prevailing jamming technique is deploying noise.

Noise jamming techniques reduce the cumulative received SNR by increasing the thermal noise level at the receiver. Barrage jamming, spot jamming and sweep jamming are the three most common types of noise jamming [21]. These noise jamming techniques, respectively, focus the transmit power on multiple frequencies at the same time, a fleet of frequencies and a single frequency. A number of jamming attacks research in radar systems have focused on the Barrage jamming where the entire bandwidth of the transmission is targeted with noise. The adverse effect of jamming has been extensively studied, as outlined in [22]. The effect of jamming in millimeter-wave (mmWave) communications has been examined in [23], [24]. For the mmWave Massive MIMO systems, a reinforcement learning based power control strategy, based on the signalto-interference-plus-noise ratio (SINR) of the signals and the jamming strength has been considered in [23], while a hybrid beamforming design to cancel the interference coming from jamming signal has been presented in [24].

In our recently published work [27], we developed a practical approach for deploying PNC in multi-user Massive MIMO systems, utilizing QPSK modulation scheme. In this Massive MIMO PNC work, we formulated a Maximum a Posteriori (MAP) based PNC mapping scheme, leveraging on the existing MIMO's linear detectors such as Zero-Forcing (ZF) and Minimum Mean Square Error (MMSE). The transmissions were over the Rayleigh fading channel with additive-white Gaussian noise (AWGN) at the receive antennas and the performance evaluation revealed that in the lower SNR regime, our PNC scheme outperformed conventional Massive MIMO in those structured noisy and interfered channels. The lingering question is now if such a robust PNC scheme can withstand uncontrolled jamming signals from a jammer.

In this article, we study a multi-user Massive MIMO system, where a base station's (BS) capacity to perform PNC is challenged by an active jammer that persistently transmits jamming signals. We investigate the error performance of the BS's capability to estimate the PNC symbols amid the active jamming signals. The contributions of this work are summarized as follows. This work:

- investigates the resilience of an existing robust PNC scheme over jamming attack in Rayleigh channels, focusing on the error performance.

- models the jamming signal using Gaussian noise, such that the jamming signal dominates the received AWGN noise at the receive antennas of the BS.

- examines jamming attacks in mmWave communications by employing the robust PNC scheme.

- evaluates and analyzes the performance of the system, using Monte-Carlo simulations, and provides perspectives on the implications of the results.

The remainder of this article is organized as follows. In Section II, we present the Massive MIMO PNC system model and considered scenarios. The channel models for both sub-6 GHz and mmWave systems are given in Section III. In Section IV, the performance analysis of Massive MIMO PNC under jamming attack is presented, followed with the simulation results in Section V. The conclusions and future research directions are given in section VI.

\section{JAMMing ATtACK TO A MASSIVE-MiMO PNC SYSTEM}

The system model is shown in Fig. 1 and it depicts a single cell Massive MIMO with centralized BS, through which the legitimate users (LUEs) communicate. The goal in this setup is for each of the LUEs to communicate and receive symbols from every other LUE. The LUEs transmit their symbols in the uplink (UL), the BS estimates the PNC symbols from the aggregated received symbols and then broadcasts the PNC symbols back in the downlink (DL) to the respective LUEs. In the same system model, there is a jammer whose objective is to sabotage the correctness of the PNC symbols estimation by sending jamming signals. The jammer has a few degrees of freedom in adversely influencing the estimation of the PNC symbols by the BS. These include the transmit power of the jamming signal and number of antennas to transmit the jamming signals. In essence, it is assumed that the jammer has multiple number of transmit antennas. The jammer's position is assumed to be outside of the cell in which the LUEs are communicating through the BS. The channel between the BS and the jammer is assumed not to be known by the BS and communications between these two entities are independent of the communications between the BS and LUEs. The jamming technique can be assumed to be any of those mentioned in Section I.

In this single cell Massive MIMO scenario, $M$ denotes the number of antennas through which the BS receives UL messages from the LUEs, $N$ denotes the number of LUEs which are equipped with $K$ number of antennas, and $L=$ $K \times N$ input antennas. As depicted in Fig. 1, the received symbols vector at the BS is given by

$$
\boldsymbol{r}=\boldsymbol{H} \boldsymbol{s}+\boldsymbol{J} z+\boldsymbol{n}
$$

where $\boldsymbol{r} \in \mathbb{C}^{M \times 1}$ denotes received symbols complex vector, $\boldsymbol{s}=\left[\begin{array}{llll}s_{1} & s_{2} & \ldots & s_{L}\end{array}\right]^{\mathrm{T}} \in \mathbb{C}^{L \times 1}$, is the transmitted symbols 


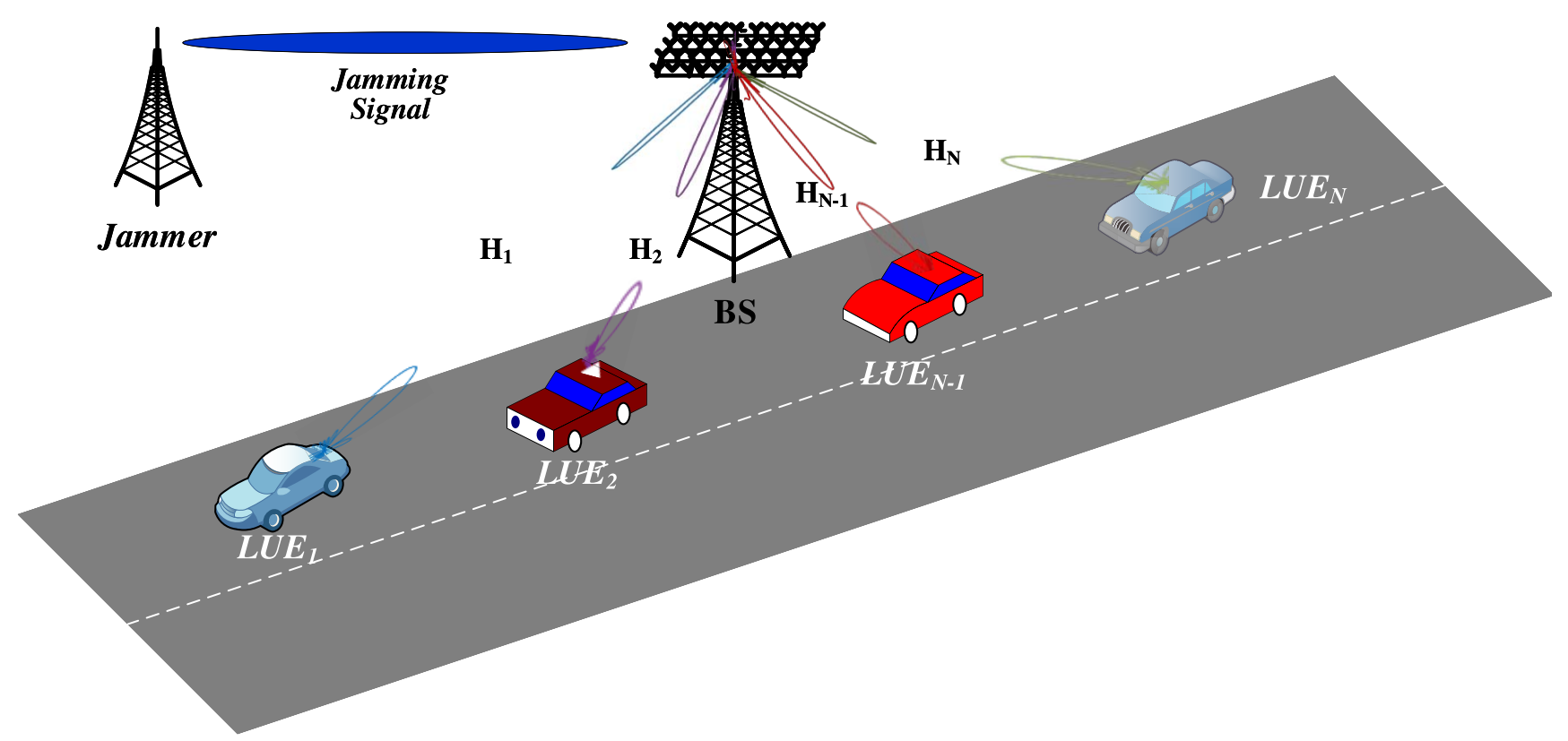

Fig. 1. A system model for a multi-user massive MIMO vehicular network under jamming attack.

complex vector from all users, $\boldsymbol{H} \in \mathbb{C}^{M \times L}$ is the complex channel matrix, $\boldsymbol{J} \in \mathbb{C}^{M \times J}$ is the jammer to BS complex channel matrix, $z \in \mathbb{C}^{J \times 1}$ is the jamming signal symbol complex vector, and $\boldsymbol{n} \in \mathbb{C}^{M \times 1}$ is independent and identically distributed (i.i.d.) AWGN with zero mean and variance, $\sigma_{n}^{2}$. We assume that the transmitted symbols are Gray-scale M-ary Quadrature Amplitude Modulation (QAM) and the average transmitted energy per symbol is $\mathbb{E}\left[\left|s_{i}\right|^{2}\right]=E_{s} / L$, where $E_{s}$ is the M-ary QAM symbol energy at each antenna.

We consider linear detectors to estimate the transmitted symbols from the received symbols at the receive antennas of the BS. Zero Forcing (ZF) and Minimum Mean Square Error (MMSE) detectors are the main focus with respect to linear detectors. They are known to have desirable computational complexity and are proven to perform well in Massive MIMO networks [27]. We also assume that there is a perfect channel state information (CSI) at the BS (i.e., the channel matrix $\boldsymbol{H}$ is known at the receiver (BS)). The estimated complex symbols vector, $\widehat{\boldsymbol{s}}$, is given as

$$
\widehat{s}=\boldsymbol{G r},
$$

where $\boldsymbol{G}$ is the complex detection matrix, which is also expressed, respectively, for ZF and MMSE, as

$$
\begin{aligned}
\boldsymbol{G}^{\mathrm{ZF}} & =\left(\boldsymbol{H}^{\mathrm{H}} \boldsymbol{H}\right)^{-1} \boldsymbol{H}^{\mathrm{H}}, \\
\boldsymbol{G}^{\mathrm{MMSE}} & =\left(\boldsymbol{H}^{\mathrm{H}} \boldsymbol{H}+\sigma_{n}^{2} \mathrm{I}\right)^{-1} \boldsymbol{H}^{\mathrm{H}},
\end{aligned}
$$

where $\sigma_{\mathrm{n}}^{2}$ is AWGN noise variance at the received antennas. As shown in (3) and (4), $\boldsymbol{G}^{\mathrm{ZF}}$ and $\boldsymbol{G}^{\mathrm{MMSE}}$ are both dependent on the complex channel matrix, $\boldsymbol{H}$.

As already stated in previous sections, in [27], we developed a Massive MIMO PNC algorithm, where a multi-antenna BS estimates PNC symbols from received interfered symbols, which are composed of superimposed transmitted symbols from multi-user multi-antenna UEs. The concept is based on a linear transformation of the channel between the BS and the UEs, using a sum-difference (SD) matrix, under the assumption that channel is known to the BS. The SD matrix ensures that the transmitted symbols are grouped into clusters of transmit antennas, from the UEs that intend to communicate with each other. The BS then estimates a sum and difference of the transmitted symbols in the cluster, from the received symbols using linear detectors such as ZF and MMSE. The detection matrix is based on the SD transformed channel matrix. Therefore, the distinction here is that for conventional Massive MIMO, the complex detection matrix is dependent on the original complex channel matrix, whereas the complex detection matrix for the Massive MIMO PNC is dependent on the transformed complex channel matrix. We refer the reader to [27] for further details of the PNC scheme.

If we consider our previous work on the Massive MIMO PNC scheme, then the system model in (1) is further expressed as

$$
\begin{aligned}
\boldsymbol{r} & =\left(\boldsymbol{H} \boldsymbol{P}_{\mathrm{sd}}^{-1}\right)\left(\boldsymbol{P}_{\mathrm{sd}} \boldsymbol{S}\right)+\boldsymbol{J} z+\boldsymbol{n} \\
& =\boldsymbol{H}_{\mathrm{sd}} \boldsymbol{S}_{\mathrm{sd}}+\boldsymbol{J} z+\boldsymbol{n},
\end{aligned}
$$

where $\boldsymbol{P}_{\mathrm{sd}}$ is the SD matrix, $\boldsymbol{H}_{\mathrm{sd}}=\frac{1}{2} \boldsymbol{H} \boldsymbol{P}_{\mathrm{sd}}$, is the linear transformation of the MIMO channel with a SD matrix, and $\boldsymbol{s}_{\mathrm{sd}}=\boldsymbol{P}_{\mathrm{sd}} \boldsymbol{s}$, clusters of SD symbols, whose estimates at the BS, are then mapped to the PNC symbols. Although the term $\boldsymbol{J} z$ in (5) is missing in the corresponding equation in [27], the estimation of the $\boldsymbol{s}_{\mathrm{sd}}$, SD symbols, also remains the same using the following equalization matrix

$$
\boldsymbol{G}_{\mathrm{sd}}=\left\{\begin{array}{l}
\left(\boldsymbol{H}_{\mathrm{sd}}{ }^{\mathrm{H}} \boldsymbol{H}_{\mathrm{sd}}\right)^{-1} \boldsymbol{H}_{\mathrm{sd}}{ }^{\mathrm{H}}, \text { for ZF } \\
\left(\boldsymbol{H}_{\mathrm{sd}}{ }^{\mathrm{H}} \boldsymbol{H}_{\mathrm{sd}}+\sigma_{n}^{2} \mathrm{I}\right)^{-1} \boldsymbol{H}_{\mathrm{sd}}{ }^{\mathrm{H}}, \text { for MMSE }
\end{array},\right.
$$

and the estimated SD symbols is given as

$$
\widehat{s}_{\mathrm{sd}}=\boldsymbol{G}_{\mathrm{sd}} \boldsymbol{r} .
$$




\section{Channel Modelling}

In this section, we will describe the channel models for both sub- $6 \mathrm{GHz}$ and mmWave frequencies.

\section{A. Sub-6 GHz Model}

The channel model for sub- $6 \mathrm{GHz}$ has been one of the well researched fields. Until recent progress in mmWave, the radio propagation in the sub- $6 \mathrm{GHz}$ was perceived as the most favorable radio propagation channel. It is still the most reliable spectrum for radio propagation, amid the scarcity of bandwidth for future wireless communication systems.

In this sub-section, we assume a multi-path propagation medium with worse-case of line-of-sight (LoS), making Rayleigh fading channel the prime focus. Given that $\boldsymbol{H}$ in (1) is assumed to be a Rayleigh fading complex channel matrix, its distribution can be modeled as a joint probability distribution of its in-phase and quadrature components. The complex entries of $\boldsymbol{H}$, i.e. $h_{i j}$, where $i$ is the receive antenna index and $j$, the transmit antenna index, are i.i.d. Gaussian random variables of zero mean and a unit variance.

The effect of the jamming signal at the BS antennas is modelled as an additive noise. In the presence of a jammer, the term $\boldsymbol{J} \boldsymbol{z}$ in (1) is assumed to dominate over the AWGN, $\boldsymbol{n}$. The signal-to-jamming plus noise ratio (SJNR) is expressed as $\operatorname{SJNR}=L \times E_{\mathrm{s}} /\left(\sigma_{\mathrm{n}}^{2}+\sigma_{\mathrm{z}}^{2}\right)$, where $\sigma_{\mathrm{z}}^{2}$ is the jamming noise variance. The signal-to-jamming ratio (SJR) is given as $\mathrm{SJR}=J \times E_{\mathrm{z}} / \sigma_{\mathrm{z}}^{2}$. In the absence of a jammer, the signal-tonoise ratio is given as, $\mathrm{SNR}=L \times E_{\mathrm{S}} / \sigma_{\mathrm{n}}^{2}$. The effect of the term $\boldsymbol{J} z$ in (6), is the prime focus of this investigation. Treating the jamming signal, $z$, as additive noise, then the lower bound of the performance of the system model is achieved since the worst case jamming signal is Gaussian when the transmitted signal is considered to be Gaussian [29]. Therefore, in our system model, the jamming term is modeled as Gaussian for sub-6 GHz. If the jamming does not dominate the AWGN, $\boldsymbol{n}$, then the decoding performance is expected to be similar to the results in our existing work [28]. Therefore, the target is to evaluate the error performance of the system model when the jamming term, $\boldsymbol{J} \boldsymbol{z}$, dominates $\boldsymbol{n}$.

\section{B. mmWave Model}

The mmWave system consists of a BS equipped with $M$ antennas having uniform linear array (ULA) antenna structure and $M$ radio frequency (RF) chains, communicating with $N$ legitimate single-antenna users in a single cell. The mmWave channel model is composed of the LoS and the non-LoS (nLoS) components [25], [26].

The channel matrix of the $n^{\text {th }}$ user, $\mathbf{H}_{n} \in \mathbb{C}^{M \times 1}$, is given as

$$
\mathbf{H}_{n}=\mathbf{H}_{n}^{\mathrm{LoS}}+\mathbf{H}_{n}^{\mathrm{nLoS}},
$$

where $\mathbf{H}_{n}^{\mathrm{LoS}}$ and $\mathbf{H}_{n}^{\mathrm{nLoS}}$, respectively, denote the LoS component and the nLos components of the mmWave channel and they are respectively, defined as

$$
\begin{aligned}
\mathbf{H}_{n}^{\mathrm{LoS}} & =\alpha_{n}^{\mathrm{LoS}} \cdot \mathbf{a}_{n}\left(\varphi_{\mathrm{LoS}}^{\mathrm{Rx}}\right) \cdot\left(\mathbf{a}_{n}\left(\varphi_{\mathrm{LoS}}^{\mathrm{Tx}}\right)\right)^{\mathrm{H}}, \\
\mathbf{H}_{n}^{\mathrm{nLoS}} & =\frac{1}{\sqrt{(C)}} \sum_{c=1}^{C} \sum_{s=1}^{S_{n, c}} \alpha_{n, c, s} \cdot \mathbf{a}_{n}\left(\varphi_{c, s}^{\mathrm{Rx}}\right) \cdot\left(\mathbf{a}_{n}\left(\varphi_{c, s}^{\mathrm{Tx}}\right)\right)^{\mathrm{H}},
\end{aligned}
$$

where $C$ and $S_{n, c}$ denote the number of clusters and the number of sub-paths in each cluster, respectively. $\alpha$ represents instantaneous complex channel gain for LoS and nLoS components. Moreover, $\varphi$ indicates the azimuth angle and $\mathbf{a}\left(\varphi^{\mathrm{Rx}}\right)$, $\mathbf{a}\left(\varphi^{\mathrm{Tx}}\right)$ respectively, denote angle of arrival (AoA) array factor at the receiver and angle of departure (AoD) array factor at the transmitter. $(\mathbf{a}(.))^{\mathrm{H}}$ denotes the complex transpose of the antenna array factor.

For the ULA, the antenna array response is defined by,

$\mathbf{a}(\varphi)=\frac{1}{\sqrt{M}}\left[1, \ldots, e^{j(m-1) k d_{x} \sin (\varphi)}, \ldots, e^{j(M-1) k d_{x} \sin (\varphi)}\right]^{T}$

where $M$ is the number of antennas in horizontal axis, $k$ is the wave number which is defined as $k=\frac{2 \pi}{\lambda}$. The inter-element spacing (distance between two adjacent antenna elements) is indicated by $d_{x}=0.5 \lambda$.

\section{Performance Analysis of Massive Mimo PNC UNDER JAMMING ATTACK}

The BER analytical analysis of Massive MIMO PNC, with transmission in Rayleigh channel, is rather tedious. Therefore, the focus of this article is to investigate the error performance analysis using numerical analysis. Before looking into the simulation results, we would like to establish the procedure in the evaluation of the BER performance of the Massive MIMO PNC, over Rayleigh channel, and under jamming attack. In (8), we established how Massive MIMO linear detection is utilized in the PNC scheme to estimate SD symbols from the received symbols, using a detection matrix based on rather a linear SD transformed channel matrix. Estimating the SD symbols from the received symbols is just a step to the final goal, which is estimating the actual PNC symbols. The estimation of the PNC is a function of the estimated SD symbols as given below

$$
\widehat{\boldsymbol{s}}_{\mathrm{PNC}}=f_{\mathrm{PNC}}\left(\widehat{\boldsymbol{s}}_{\mathrm{Sd}}\right),
$$

where $\widehat{\boldsymbol{s}}_{\mathrm{PNC}}$ is the estimated PNC symbols, $f_{\mathrm{PNC}}($.$) , the$ PNC estimation function and $\widehat{\boldsymbol{s}}_{\mathrm{sd}}$, the estimated SD symbols. Maximum a posteriori (MAP) estimation is a well known Bayesian inference estimator that can be used to estimate an unknown parameter, given an observation. To estimate the PNC symbols, given the SD symbols, MAP estimator is used. Assuming $\theta$ is the unknown to be determined from the observation, $x$, then MAP estimator is given as, the $\theta$ that maximizes the posteriori, as expressed below

$$
\begin{aligned}
\widehat{\theta}_{\mathrm{MAP}} & =\underset{\theta}{\operatorname{argmax}} P(\theta \mid x) \\
& \approx \underset{\theta}{\operatorname{argmax}} P(x \mid \theta) P(\theta),
\end{aligned}
$$

where $P(\theta \mid x)$ is the posteriori or the conditional probability of the unknown, $\theta$, given the observation, $x$, whereas $P(x \mid \theta)$ is the likelihood or the conditional probability of the observation given the unknown, and $P(\theta)$ and $P(x)$ are respectively, the marginal distribution functions for the unknown and the observation. If $P(\theta)$ is assumed to be equally likely, then 
the MAP estimator approximates to Maximum Likelihood Estimator (MLE) as follows

$$
\widehat{\theta}_{\text {MAP }} \approx \underset{\theta}{\operatorname{argmax}} P(x \mid \theta)=\widehat{\theta}_{\text {MLE }} .
$$

Having defined the MAP decoder, we can now employ it in our PNC Mapping scheme in Massive MIMO systems in estimating the NC symbols at the physical layer. In [28], clusters of estimated SD symbols that are mapped to PNC symbols are expressed as $\left\{\left(\widehat{s}_{\mathrm{sd}_{\mathrm{i}}}, \widehat{s}_{\mathrm{sd}, \mathrm{Q}+\mathrm{i}}\right) \mid 1 \leq i \leq Q\right\}$. We assume that the corresponding set of clusters of transmitted input symbol, $\left\{\left(s_{i}, s_{Q+i}\right) \mid 1 \leq i \leq Q, s_{i}, s_{Q+i} \in \Theta\right\}$, are modulated symbols from the constellation, represented as $\Theta$. Then, the Likelihood of obtaining the PNC symbol, $s_{i} \widehat{\oplus s_{Q}+i}$, such that $s_{i} \widehat{\oplus s_{Q}+i} \in \Theta$ is expressed as

$$
\begin{aligned}
& L\left(s_{i} \widehat{\oplus s_{Q}+i}=v \mid \widehat{s}_{\mathrm{sd}_{\mathrm{i}}}, \widehat{s}_{\mathrm{sd}, \mathrm{Q}+\mathrm{i}}\right) \\
& =\sum_{v \in \Theta} P\left(\widehat{s}_{\mathrm{sd}_{\mathrm{i}}}, \widehat{s}_{\mathrm{sd}_{, \mathrm{Q}+\mathrm{i}}} \mid s_{i} \widehat{\oplus s_{Q}+i}=v\right) \\
& =\sum_{v \in \Theta} P\left(\widehat{s}_{\mathrm{sd}, \mathrm{i}} \mid s_{i} \widehat{\oplus s_{Q}+i}=v\right) P\left(\widehat{s}_{\mathrm{sd}, \mathrm{Q}+\mathrm{i}} \mid s_{i} \widehat{\oplus s_{Q}+i}=v\right) \\
& =\sum_{v \in \Theta} P\left(\widehat{s}_{\mathrm{sd}_{\mathrm{i}}} \mid \bar{s}_{\mathrm{sd}_{\mathrm{i}}}^{(v)}\right) P\left(\widehat{s}_{\mathrm{sd}_{, \mathrm{Q}+\mathrm{i}}} \mid \bar{s}_{\mathrm{sd}, \mathrm{Q}+\mathrm{i}}^{(v)}\right),
\end{aligned}
$$

where $\bar{s}_{\mathrm{sd}_{\mathrm{i}}}^{(v)}$ and $\bar{s}_{\mathrm{sd}, \mathrm{Q}+\mathrm{i}}^{(v)}$, are respectively, the expected summation and difference symbols, mapped to the PNC symbol, $v \in \Theta$. Since the noise in (1) and (6) is assumed to be AWGN, the likelihood function can be assumed to be Gaussian. Using the Gaussian probability density function, (17) can be expressed further as

$$
\begin{gathered}
L\left(s_{i}{\widehat{\oplus s_{Q+i}}}=v \mid \widehat{s}_{\mathrm{sd}_{\mathrm{i}},} \widehat{s}_{\mathrm{sd}, \mathrm{Q}+\mathrm{i}}\right) \\
=\sum_{v \in \Theta} \frac{1}{2 \pi \sqrt{\sigma_{i}^{2} \sigma_{Q+i}^{2}}} e^{-\frac{\left(\widehat{s}_{\mathrm{sd}_{\mathrm{i}} \mathrm{i}}-\bar{s}_{\mathrm{sd}_{\mathrm{i}}}^{(v)}\right)^{2}}{2 \sigma_{i}^{2}}} \\
\quad e^{-\frac{\left(\widehat{s}_{\mathrm{sd}, \mathrm{Q}+\mathrm{i}}-\bar{s}_{\mathrm{sd}_{\mathrm{Q}+\mathrm{i}}}^{(v)}\right)^{2}}{2 \sigma_{Q+i}^{2}}},
\end{gathered}
$$

where $\sigma_{i}^{2}, \sigma_{Q+i}^{2}$ are the noise variances of the $i^{t h}$ and $\{Q+i\}^{\text {th }}$ streams, respectively. Knowing the general likelihood's function for the set of all clusters, $L\left(s_{i} \widehat{\oplus s_{Q}+i}=\right.$ $\left.v \mid \widehat{s}_{\mathrm{sd}, \mathrm{i}}, \widehat{s}_{\mathrm{sd}, \mathrm{Q}+\mathrm{i}}\right)$, the MAP estimator in (16) can be employed to determine the PNC symbol of each cluster as follows

$$
\begin{aligned}
& \left(s_{i} \widehat{\oplus s_{Q}+i}\right)_{\mathrm{MAP}}^{(v)} \\
& \quad \underset{s_{i} \oplus s_{Q+i}}{\operatorname{argmax}} L\left(s_{i} \widehat{\oplus s_{Q}+i}=v \mid \widehat{s}_{\mathrm{sd}_{, i}}, \widehat{s}_{\mathrm{sd}, \mathrm{Q}+\mathrm{i}}\right) P\left(\widehat{s}_{\mathrm{sd}_{\mathrm{i}}}, \widehat{s}_{\mathrm{sd}_{, \mathrm{Q}+\mathrm{i}}}\right) .
\end{aligned}
$$

The overall set of estimated PNC symbols is expressed as

$$
\begin{aligned}
\widehat{s}_{\mathrm{PNC}}^{i} & =f_{\mathrm{PNC}}\left(\widehat{s}_{\mathrm{Sd}_{\mathrm{i}}}, \widehat{s}_{\mathrm{Sd}_{, \mathrm{Q}+\mathrm{i}}}\right) \\
& =\left\{\left(s_{i} \widehat{\oplus s_{Q}+i}\right)_{\mathrm{MAP}}^{(v)} \mid 1 \leq i \leq Q, \quad v \in \Theta\right\} .
\end{aligned}
$$

\section{Simulation Results}

In this section, we present the simulation results for both the sub- $6 \mathrm{GHz}$ and mmWave systems.
TABLE I

SimUlation PARAMETERS

\begin{tabular}{|c|c|}
\hline Parameters & Values \\
\hline No. antennas at BS, $M$ & $4, \ldots, 16$ \\
\hline No. antennas per UE, $K$ & 2 \\
\hline No. UEs, $N$ & $2, \ldots, 8$ \\
\hline Channel, $H$ & i.i.d Rayleigh \\
\hline SNR [dB] & $0, \ldots, 50$ \\
\hline Modulation & QPSK, 16QAM \\
\hline Packet size per UE & 100 modulated symbols \\
\hline No. iterations & $10^{4}$ \\
\hline Channel Coding & Uncoded \\
\hline Linear Detectors & ZF, MMSE \\
\hline No. antennas at Jammer, $J$ & $\{1,2\}$ \\
\hline SJR [dB] & $10, \ldots, 20$ \\
\hline
\end{tabular}

\section{A. In Sub-6 GHz}

In this subsection, we evaluate the error performance of MIMO PNC scheme against jamming attack in sub-6GHz frequencies. The simulation parameters are listed in Table I. In the simulation, we have a BS with multiple number of antennas communicating with LUEs, and for simplicity, we assume that the number of antennas at each LUE is the same. The jammer is also assumed to have multiple number of antennas and transmitting independently of the LUEs. The objective of the BS is to perform PNC employing the algorithm in [28], whereas, that of the jammer is to introduce errors in the PNC algorithm execution. In the simulation setup, the jammer has a couple of possibilities to influence the performance of the BS. We simulated various scenarios that include conventional MIMO without PNC, MIMO with PNC, with and without jamming attack. We regulated the transmit power and the number of antennas of the jammer, and then, countered it with regulating the dimension of the received antennas of the BS and also the modulation scheme. cases of MIMO and PNC and the jammer.

Fig. 2 presents the error performance for all use cases when the modulation scheme is QPSK, employing both ZF and MMSE. The BS has four antennas, and each of the two UEs has two antennas and the jammer has a single antenna with SJR of $10 \mathrm{~dB}$. Although the dimension of the antennas at the BS in this setup is not Massive MIMO, its worth providing result for completeness. In the simulation result, the BER of MIMO and PNC is comparably better in all use cases. The BER of the MMSE-based use cases performed better than the ZF counterpart, which is as expected, as ZF does not consider noise at the receive antennas in the detection of the transmitte symbols. Among the use cases, it can be seen that non-jammed MIMO and PNC performed better than the nonjammed, but only MIMO. This shows that MIMO and PNC can coexist without any detriment to the performance of the former. It can also be seen that both jammed MIMO and 


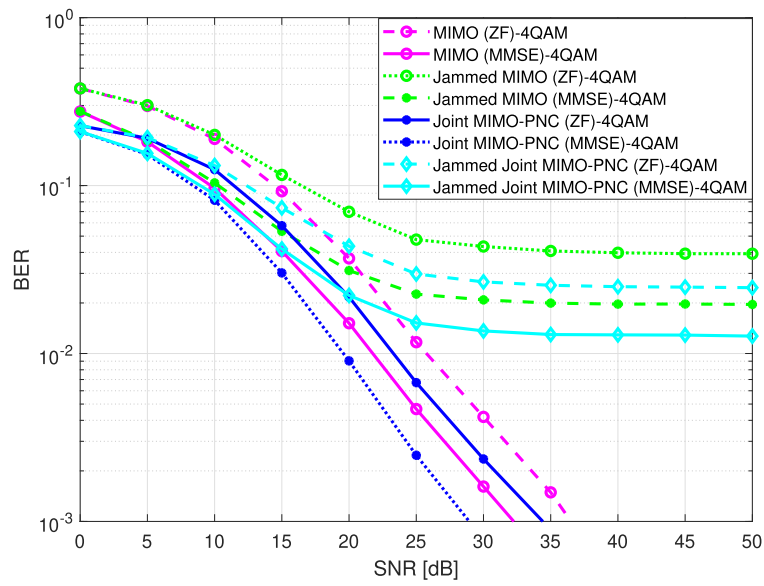

Fig. 2. BER performance comparison between i) conventional multi-user MIMO ii) multi-user MIMO with jamming, iii) multi-user MIMO with PNC and iv) multi-user MIMO with PNC and jamming, with $10 \mathrm{~dB}$ jamming SJR, for $M=4, N=2, K=2$ and $J=1$

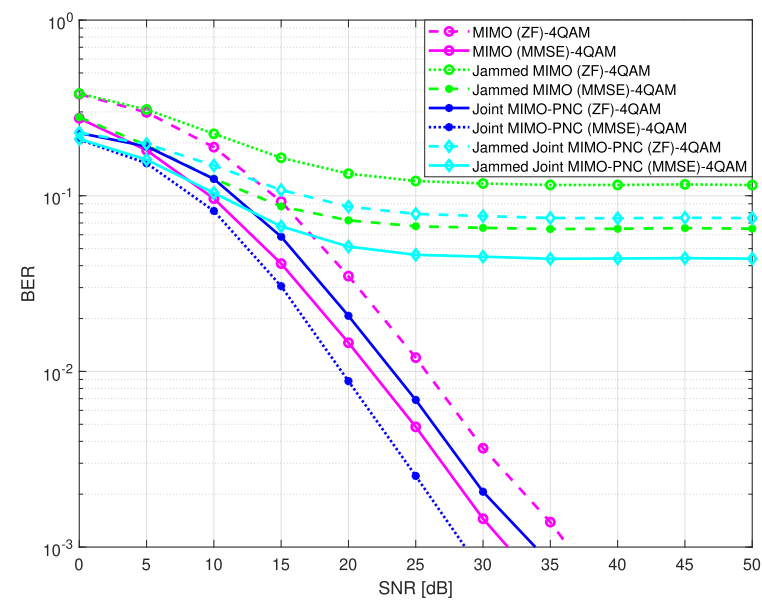

Fig. 3. BER performance comparison between i) conventional multi-user MIMO ii) multi-user MIMO with jamming, iii) multi-user MIMO with PNC and iv) multi-user MIMO with PNC and jamming, with $10 \mathrm{~dB}$ jamming SJR, for $M=4, N=2, K=2$ and and $J=2$.

jammed MIMO PNC performed comparatively close to the non-jammed counterpart, until at about $15 \mathrm{~dB}$ SNR. Further from this SNR, the jammed BER for the usecases starts to remain steady. This is an indication of the PNC scheme performing well against jamming in the lower SNR region, and this is achieved at twice the spectral efficiency.

In Fig. 3, utilizing the same setup as Fig. 2, we doubled the number of antennas of the jammer. The simulation result revealed a degrade in the former compared to the latter. This is an indication that by increasing the number of antennas of the jammer, the jamming signal adversely influences the decoding capabilities of the BS. The jammer may not want to expend extra cost in its quest of impairing the decoding capabilities of the BS, by increasing the number of transmit antennas.

We increased the number of antennas at the BS to sixteen in Fig. 4, with the rest of the simulation parameters remaining the same as in Fig. 2. The simulation results of the former

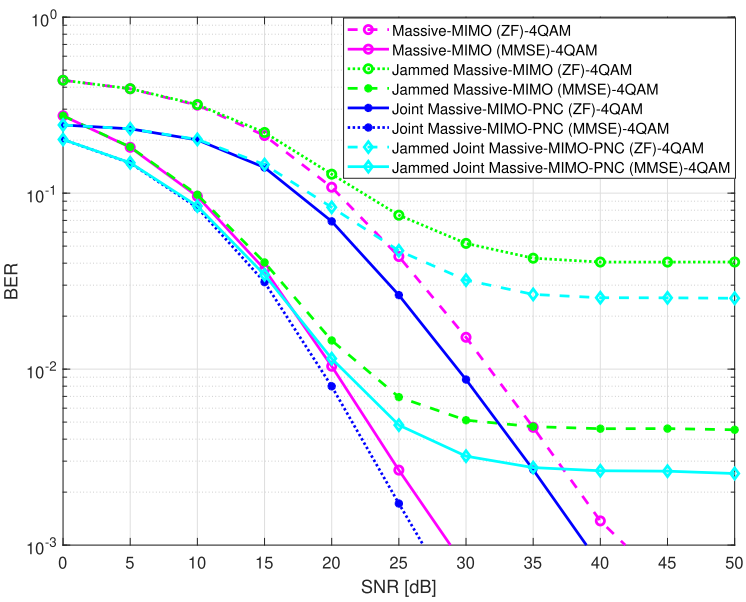

Fig. 4. BER performance comparison between i) conventional multi-user MIMO ii) multi-user MIMO with jamming, iii) multi-user MIMO with PNC and iv) multi-user MIMO with PNC and jamming, with 10dB jamming SJR, for $M=16, N=8, K=2$ and $J=1$

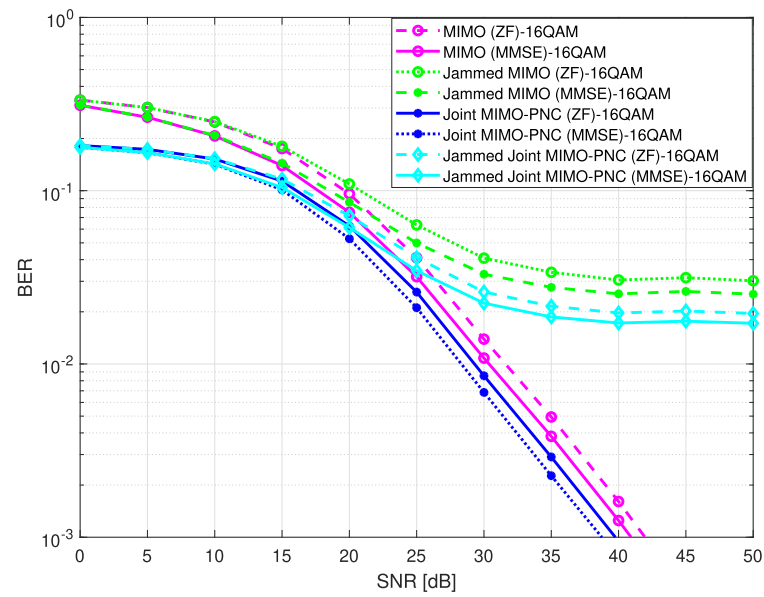

Fig. 5. BER performance comparison between i) conventional multi-user MIMO ii) multi-user MIMO with jamming, iii) multi-user MIMO with PNC and iv) multi-user MIMO with PNC and jamming, with $10 \mathrm{~dB}$ jamming SJR, for $M=4, N=2, K=2$ and $J=1$.

revealed a similar pattern to that of the latter, except the BER starts to remain steady after $30 \mathrm{~dB}$ SNR. This is an indication that the more antennas the BS has, the better the resilience against a jamming attack it has in the lower SNR regions.

In Fig. 5, we repeated the setup in Fig. 2 and Fig. 3, respectively, except deploying a higher-order modulation scheme of 16-QAM. The result showed a similar pattern to the QPSK counterpart, except the performance is better, as the BER starts to remain steady at higher SNR, an indication that PNC performs better in the lower SNR regions against jamming attack.

\section{B. In mmWave}

In this subsection, we evaluate the BER performance in the mmWave communications under jamming attacks by employing PNC based on linear detection, which has lower complexity. The mmWave channel parameters described in 


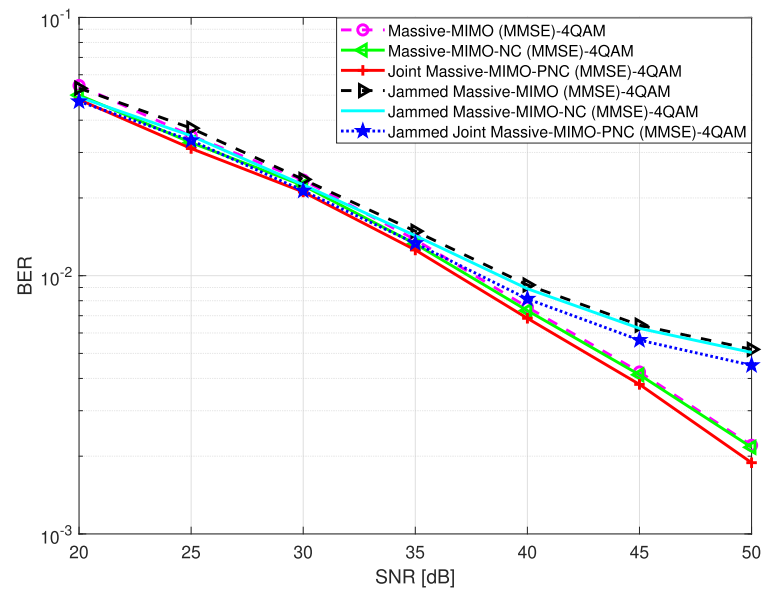

Fig. 6. BER performance comparison of mmWave communications for different schemes under jamming attack, with $30 \mathrm{~dB}$ jamming SJR, for $M=16$, $N=16, K=1$ and $J=1$.

[25] and [26] are used in our mmWave system simulation, which includes one BS having $M=16$ antennas and communicating with $N=16$ legitimate users. The simulation results are shown in Fig. 6. It is observed that the mmWave communications by employing PNC through linear detection provides much better performance compared to the conventional mmWave schemes.

\section{CONCLUSION}

In this work, we investigated the resilience of Massive MIMO PNC to jamming attack in both sub-6 $\mathrm{GHz}$ and mmWave. In our previous work, we showed how the combination of Massive MIMO and PNC does not impact the BER any worse than it would in Massive MIMO system without PNC. Infact, our results revealed that the BER of Massive MIMO and PNC is slightly better than the BER of only Massive MIMO. Massive MIMO is generally resilient to jamming attack due its high degree of freedom. We investigated our previous work on Massive MIMO and PNC against a barraging attack from a jammer, when the jammer is able to use any number of transmit antennas. Over Rayleigh fading channels, our simulation results revealed that Massive MIMO PNC performs better in lower SNRs to jamming attacks, as opposed to the conventional Massive MIMO system, and at twice the spectral efficiency. In addition to that, for mmWave communications under jamming attack, the MIMO-PNC system still provided acceptable BER performance.

\section{REFERENCES}

[1] S. Zhang, S. C. Liew, and P. P. Lam, "Physical layer network coding," in Proc. 12th Ann. Int. Conf. Mobile Commun. Net (MOBICOM), Los Angeles, CA, USA, Sep. 2006, pp. 358-365.

[2] S. C. Liew, S. Zhang, and L. Lu, "Physical-layer network coding: Tutorial, survey, and beyond," Phys. Commun., vol. 6, pp. 4-42, Mar. 2013.

[3] A. Burr and D. Fang, "Linear physical-layer network coding for $5 \mathrm{G}$ radio access networks," in Proc. 1st Int. Conf. 5 G Ubiquitous Connectivity, Akaslompolo, Finland, 2014, pp. 116-221.

[4] S. Zhang and S. C. Liew, "Physical layer network coding with multiple antennas," in Proc. IEEE Wireless Commun. Netw. Conf., Apr. 2010, pp. 1-6.
[5] D. H. Vu and X. N. Tran, "Physical network coding for bidirectional relay MIMO-SDM system," in Proc. Int. Conf. Adv. Technol. Commun., Oct. 2013, pp. 141-146.

[6] T. Yang, X. Yuan, L. Ping, I. B. Collings, and J. Yuan, "Eigendirection alignment based physical-layer network coding for MIMO two-way relay channels," in Proc. IEEE Int. Symp. Inf. Theory (ISIT), SaintPetersburg, Russia, Jul. 2011, pp. 2253-2257.

[7] T. Yang, X. Yuan, L. Ping, I. B. Collings, and J. Yuan, "A new physical-layer network coding scheme with eigen-direction alignment precoding for MIMO two-way relaying," IEEE Trans. Commun., vol. 61, no. 3, pp. 973-986, Mar. 2013.

[8] R. Zhang, Y.-C. Liang, C. C. Chai, and S. Cui, "Optimal beamforming for two-way multi-antenna relay channel with analogue network coding," IEEE J. Sel. Areas Commun., vol. 27, no. 5, pp. 699-712, Jun. 2009.

[9] R. Annavajjala, A. Maaref, and J. Zhang, "Multiantenna analog network coding for multihop wireless networks," Int. J. Digit. Multimedia Broadcast., vol. 2010, pp. 1-10, 2010.

[10] Z. Ding, I. Krikidis, J. Thompson, and K. K. Leung, "Physical layer network coding and precoding for the two-way relay channel in cellular systems," IEEE Trans. Signal Process., vol. 59, no. 2, pp. 696-712, Feb. 2011.

[11] S. C. Liew, S. Zhang, and L. Lu, "Physical-layer network coding: Tutorial, survey, and beyond," Phys. Commun., vol. 6, pp. 4-42, Mar. 2013.

[12] M. Huang, J. Yuan, and T. Yang, "Error probability of physical-layer network coding in multiple-antenna two-way relay channel," in Proc. IEEE Global Commun. Conf. (GLOBECOM), Anaheim, CA, USA, Dec. 2012, pp. 4725-4730.

[13] M. Huang and J. Yuan, "Physical-layer network coding with alamouti scheme for the TWRC with linear decoder," in Proc. Austral. Commun. Theory Workshop (AusCTW), Sydney, NSW, Australia, Feb. 2014, pp. 108-113.

[14] M. Huang and J. Yuan, "Error performance of physical-layer network coding in multiple-antenna TWRC," IEEE Trans. Veh. Technol., vol. 63, no. 8, pp. 3750-3761, Oct. 2014.

[15] S. Zhang, Q. F. Zhou, C. Kai, and W. Zhang, "Full diversity physical-layer network coding in two-way relay channels with multiple antennas," IEEE Trans. Wireless Commun., vol. 13, no. 8, pp. 4273-4282, Aug. 2014.

[16] J. S. Lemos and F. A. Monteiro, "Full-duplex massive MIMO with physical layer network coding for the two-way relay channel," in Proc. IEEE Sensor Array Multichannel Signal Process. Workshop (SAM), Jul. 2016, pp. 1-5.

[17] T. Peng, Y. Wang, A. G. Burr, and M. S. Bahaei, "A physical layer network coding design for 5G network MIMO," in Proc. IEEE Global Commun. Conf. (GLOBECOM), Abu Dhabi, United Arab Emirates, Dec. 2018, pp. 1-7.

[18] T. Peng, Y. Wang, A. G. Burr, and M. R. Shikh-Bahaei, "Physical layer network coding in network MIMO: A new design for 5G and beyond," IEEE Trans. Commun., vol. 67, no. 3, pp. 2024-2035, Mar. 2019.

[19] A. Burr and D. Fang, "Linear physical-layer network coding for 5G radio access networks," in Proc. 1st Int. Conf. 5 G for Ubiquitous Connectivity, 2014, pp. 116-121.

[20] Y. Zou, J. Zhu, X. Wang, and L. Hanzo, "A survey on wireless security: Technical challenges, recent advances, and future trends," Proc. IEEE, vol. 104, no. 9, pp. 1727-1765, Sep. 2016.

[21] A. Mpitziopoulos, D. Gavalas, C. Konstantopoulos, and G. Pantziou, "A survey on jamming attacks and countermeasures in WSNs," IEEE Commun. Surveys Tuts., vol. 11, no. 4, pp. 42-56, 4th Quart., 2009.

[22] J. Gao, S. A. Vorobyov, H. Jiang, and H. V. Poor, "Worst-case jamming on MIMO Gaussian channels," IEEE Trans. Signal Process., vol. 63, no. 21, pp. 5821-5836, Nov. 2015.

[23] Z. Xiao, B. Gao, S. Liu, and L. Xiao, "Learning based power control for mmWave massive MIMO against jamming," in Proc. IEEE Global Commun. Conf. (GLOBECOM), Dec. 2018, pp. 1-6.

[24] J. Zhu, Z. Wang, Q. Li, H. Chen, and N. Ansari, "Mitigating intended jamming in mmWave MIMO by hybrid beamforming," IEEE Wireless Commun. Lett., vol. 8, no. 6, pp. 1617-1620, Dec. 2019.

[25] M. K. Samimi and T. S. Rappaport, "3-D millimeter-wave statistical channel model for 5G wireless system design," IEEE Trans. Microw. Theory Techn., vol. 64, no. 7, pp. 2207-2225, Jul. 2016.

[26] I. A. Hemadeh, K. Satyanarayana, M. El-Hajjar, and L. Hanzo, "Millimeter-wave communications: Physical channel models, design considerations, antenna constructions, and link-budget," IEEE Commun. Surveys Tuts., vol. 20, no. 2, pp. 870-913, 2nd Quart., 2018. 
[27] B. Okyere, L. Musavian, and R. Mumtaz, "Multi-user massive MIMO and physical layer network coding," in Proc. IEEE Globecom Workshops (GC Wkshps), Dec. 2019, pp. 1-6.

[28] L. Sanguinetti, E. Björnson, and J. Hoydis, "Towards massive MIMO 2.0: Understanding spatial correlation, interference suppression, and pilot contamination," 2019, arXiv:1904.03406. [Online]. Available: http://arxiv.org/abs/1904.03406

[29] S. N. Diggavi and T. M. Cover, "The worst additive noise under a covariance constraint," IEEE Trans. Inf. Theory, vol. 47, no. 7, pp. 3072-3081, Nov. 2001.

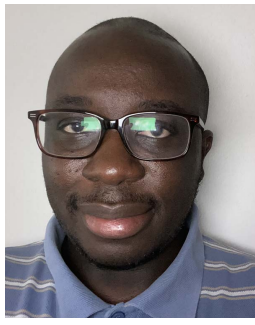

Bismark Okyere (Graduate Student Member, IEEE) received the B.Eng degree (Hons.) in electronic engineering (majoring in telecommunications) from Multimedia University, Malaysia, in 2006, the double M.Sc. degree in electrical engineering and information technology from the Karlsruhe Institute of Technology, Germany, and also from the Politecnico di Torino, Italy, in 2009. He is currently pursuing the $\mathrm{Ph} . \mathrm{D}$. degree in electronic systems engineering with the University of Essex, U.K. His research interests include network coding, massive MIMO, and physical layer security.

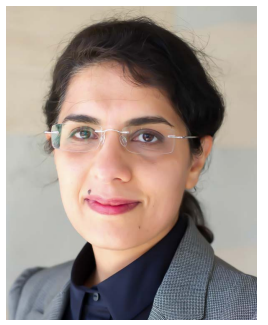

Leila Musavian (Member, IEEE) received the Ph.D. degree in telecommunications from Kings College London, U.K. She was a Post-Doctoral Fellow with INRS-EMT, Canada, from 2006 to 2008, a Research Associate with Loughborough University, U.K., from 2009 to 2010, and a Research Associate with McGill University from 2011 to 2012. She was a Lecturer with InfoLab21, Lancaster University, from 2012 to 2016. She is currently a Deputy ProVice-Chancellor for research with the University of Essex and also a Reader in telecommunications with the School of Computer Science and Electronic Engineering. Her research interests include radio resource management for low latency communications, B5G/5G, mmWave communications, massive MIMO, and energy harvesting communications.

She has been a TPC member of several conferences including IEEE ICC, IEEE GLOBECOM, IEEE WCNC, IEEE ICCCN, IEEE PIMRC, and ChinaCom. She has been a Lead Chair UHS5G WP in IEEE Globecom 2018, UHSLLS WP in IEEE WCNC 2019, a Lead Chair of URLLC Special Session in IEEE PIMRC 2018, a TPC Co-Chair of CorNer 2016 (in conjunction with ISWCS 2016), and a Co-Chair of mmWave 5G (STEMCOM 2016). She is currently the Workshop Co-Chair of VTC-Spring-2020 and the Wireless Communications Symposium Lead Co-Chair of IEEE ICC 2021. She was an Associate Editor of Internet Technology Letters. She has been an Executive Editor of Transactions on Emerging Telecommunications Technologies from 2016 to 2019. She is an Editor of the IEEE TRANSACTIONS OF WIRELESS COMMUNICATIONS.

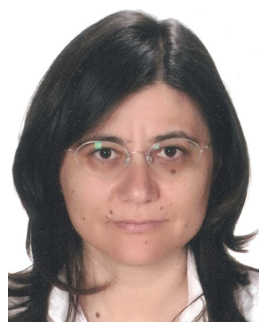

Berna Özbek (Senior Member, IEEE) is currently an Associate Professor in telecommunication with the Electrical and Electronics Engineering Department, Izmir Institute of Technology, Turkey, and also working in the field of wireless communication systems for more than 15 years. She has been awarded as a Marie-Curie Intra-European (EIF) Fellow by European Commission for two years in the project entitled Interference Management Techniques for Multicell Networks in 2010. She has coordinated one international and four national projects, served as a consultant for three Eureka-Celtic projects and two national industry driven projects. Under her supervision, 13 master's thesis and two Ph.D. dissertations have been completed. She is currently supervising two Ph.D. and two master's students and conducting one international project under Horizon2020-MCRISE programme from 2018 to 2022. She has published more than 90 peerreviewed articles, one book, one book chapter, and two patents. She is serving as a referee for several international journals, on numerous TPCs for IEEE sponsored conferences, European Commission, Turkish Republic of Ministry of Trade and Industry, and The Scientific and Technological Research Council of Turkey. Her research interests include interference management, resource allocation, limited feedback links, device-to-device communications, physical layer security, massive MIMO systems, and mmWave communications.

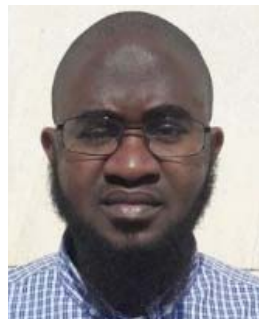

Sherif A. Busari received the B.Eng. and M.Eng. degrees in electrical and electronics engineering from the Federal University of Technology Akure (FUTA), Nigeria, in 2011 and 2015, respectively, and an industry-driven Ph.D. degree in telecommunications engineering from the Universidade de Aveiro, Portugal, in 2020. His research interests focus on technology enablers and system level simulation methodologies for $5 \mathrm{G}$ and beyond-5G networks.

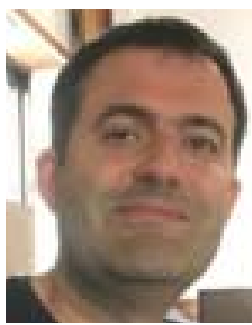

Jonathan Gonzalez received the M.Sc. and Ph.D degrees in telecommunications from the University of Surrey, U.K., in 1999 and 2004, respectively. He then became a Senior Researcher with the University of Surrey, where he was responsible for project development and research on mobile systems. He became an Honorary Senior Researcher with the University of Bradford in 2019. In 2011, he founded GS-Lda, Portugal, targeting R\&I on next generation mobile platforms. He has more than 15 years Research and Development experience in mobile communications and practical experimentation. His research interests include simulation methodologies and radio resource management. 